\title{
OPEN Identification and genetic diversity analysis of a male-sterile gene (MS1) in Japanese cedar (Cryptomeria japonica D. Don)
}

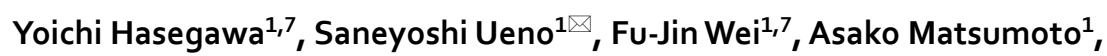
Kentaro Uchiyama ${ }^{1}$, Tokuko Ujino-Ihara ${ }^{1}$, Tetsuji Hakamata ${ }^{2}$, Takeshi Fujino ${ }^{3}$, Masahiro Kasahara ${ }^{3}$, Takahiro Bino ${ }^{4}$, Katsushi Yamaguchi ${ }^{4}$, Shuji Shigenobu ${ }^{4}$, Yoshihiko Tsumura ${ }^{5}$ \& Yoshinari Moriguchi ${ }^{6 \bowtie}$

Identifying causative genes for a target trait in conifer reproduction is challenging for species lacking whole-genome sequences. In this study, we searched for the male-sterility gene (MS1) in Cryptomeria japonica, aiming to promote marker-assisted selection (MAS) of male-sterile C. japonica to reduce the pollinosis caused by pollen dispersal from artificial $C$. japonica forests in Japan. We searched for mRNA sequences expressed in male strobili and found the gene CJt020762, coding for a lipid transfer protein containing a 4-bp deletion specific to male-sterile individuals. We also found a 30-bp deletion by sequencing the entire gene of another individual with the $m s 1$. All nine breeding materials with the allele $m s 1$ had either a 4-bp or 30-bp deletion in gene CJt020762, both of which are expected to result in faulty gene transcription and function. Furthermore, the 30-bp deletion was detected from three of five individuals in the Ishinomaki natural forest. From our findings, CJt020762 was considered to be the causative gene of MS1. Thus, by performing MAS using two deletion mutations as a DNA marker, it will be possible to find novel breeding materials of $C$. japonica with the allele $m s 1$ adapted to the unique environment of each region of the Japanese archipelago.

The breeding of forest trees is not as advanced as that of food crops and the planting trees are genetically close to individual trees of natural forests. Recent progress in genome analysis technology has promoted genome-based breeding techniques in tree plantations ${ }^{1}$. However, the construction of whole-genome sequences in conifers, which are important plantation trees, has been delayed due to the large size of their genome $(>10 \mathrm{~Gb})$ and extensive repetitive elements ${ }^{2}$. It is, therefore, challenging to identify the causative genes for a given target breeding trait in conifer species.

Cryptomeria japonica D. Don (Cupressaceae) covers over 4.5 million hectares, accounting for $44 \%$ of all Japanese artificial forests ${ }^{3}$. As a result, in 2008, 26.5\% of Japanese residents had an allergy to C. japonica pollen ${ }^{4}$. Pollinosis caused by Japanese cedar, C. japonica, is a widespread social problem in Japan. Genetically malesterile $C$. japonica trees are expected to play an important role in reducing the amount of dispersed pollen. In general, the frequency of male-sterile C. japonica trees is low within the Japanese population: for example, Igarashi et al. found two male-sterile trees among 8,700 trees in a 19 ha artificial forest ${ }^{5}$. To date, 23 genetically male-sterile C. japonica trees have been identified in Japan ${ }^{6}$. Based on the results of test crossings, four recessive male-sterile genes, MS1, MS2, MS3 and MS4, have been identified ${ }^{6-9}$ and they were mapped on linkage map ${ }^{10}$. Out of these genes, MS1 is the most frequent in $C$. japonica male-sterility breeding materials, with 11 malesterile trees homozygous for MS1 ( $\mathrm{ms} 1 / \mathrm{ms} 1)$ and five male-fertile trees heterozygous for $M S 1(\mathrm{Ms} 1 / \mathrm{ms} 1)$ found

\footnotetext{
${ }^{1}$ Department of Forest Molecular Genetics and Biotechnology, Forestry and Forest Products Research Institute, Forest Research and Management Organization, Tsukuba, Ibaraki, Japan. ${ }^{2}$ Forestry and Forest Products Research Center, Shizuoka Prefectural Research Institute of Agriculture and Forestry, Hamamatsu, Shizuoka, Japan. ${ }^{3}$ Graduate School of Frontier Sciences, The University of Tokyo, Kashiwa, Chiba, Japan. ${ }^{4}$ National Institute for Basic Biology, Okazaki, Aichi, Japan. ${ }^{5}$ Faculty of Life and Environmental Sciences, University of Tsukuba, Tsukuba, Ibaraki, Japan. ${ }^{6}$ Graduate School of Science and Technology, Niigata University, Niigata, Niigata, Japan. ${ }^{7}$ These authors contributed equally: Yoichi Hasegawa and Fu-Jin Wei. ${ }^{\square}$ email: saueno@ffpri.affrc.go.jp; chimori@agr.niigata-u.ac.jp
} 


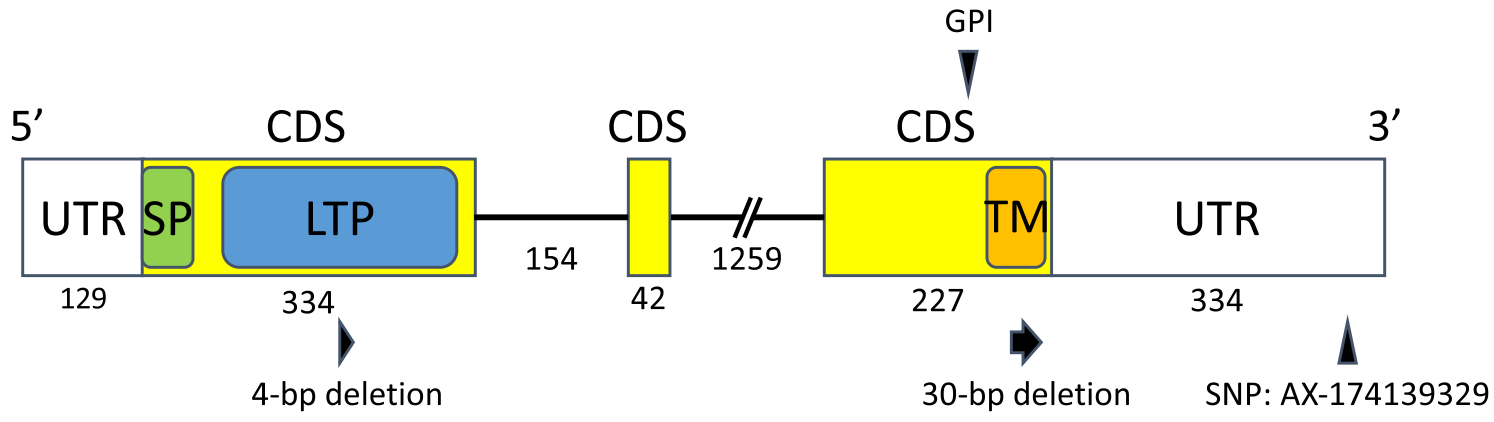

Figure 1. Schematic representation of CJt020762 including the two untranslated regions (UTRs), the three coding sequence (CDS) regions and two introns. The numbers indicate the length of nucleotide sequences in each region. LTP, predicted plant lipid transfer protein domain; SP, signal peptide; TM, transmembrane domain; GPI, potential modification site of GPI (glycosylphosphatidylinositol) anchor domain. Two deletion mutations were found on CJt020762. AX-174139329 indicates the SNP marker located at $0.0 \mathrm{cM}$ from MS1 on the linkage map constructed for the 'Fukushima-1' $\times$ 'Ooi-7' family (Hasegawa et al. ${ }^{22}$ ).

in Japan ${ }^{5,6,11-18}$. The C. japonica trees that are homozygous for $m s 1$ become male-sterile due to the failure of exine development during microspore formation ${ }^{16,19}$, but it remains unclear whether MS1 is caused by a single genetic mutation event. Additionally, there is no information about the geographical distribution of the genetic mutation responsible for MS1.

In recent years, the tightly linked DNA markers to perform marker-assisted selection (MAS) of individuals with MS1 have been developed ${ }^{20-25}$. Mishima et al. suggested reCj19250 (the DEAD-box RNA helicase gene) as a candidate gene for MS1 in C. japonica ${ }^{23}$. However, 'Ooi-7' heterozygous for MS1 could not be selected with two SNP markers contained in reCj19250 22, suggesting that reCj19250 was not the causative gene of MS1. Thus, to efficiently select individuals with $m s 1$ using MAS from various $C$. japonica populations, it is essential to search for genetic mutations that can consistently explain the MS1 phenotype.

For the MAS of C. japonica trees that are homozygous or heterozygous for MS1 and the breeding of malesterile $C$. japonica trees adapted to the unique environments in each region of the Japanese archipelago, it is important to elucidate the diversity of genetic mutations responsible for MS1 and the phylogenetic origin of MS1. Thus, in this study, we conducted the following analyses: (1) to identify the genetic mutation specific to homozygous or heterozygous individuals for $M S 1$ from mRNA sequences expressed in male strobili of $C$. japonica; (2) the functional annotation of candidate genes and deletion mutations causing $m s 1$; and (3) construct the haplotype network and haplotype map of $M S 1$ candidate genes using breeding materials with $m s 1$ and trees from natural forests on the Japanese archipelago.

\section{Results}

RNA sequencing in the coding region of CJt020762. RNA-sequencing (RNA-Seq) data were analysed to identify the genetic variation specific to individuals with the recessive allele (ms 1$)$ of $M S 1$ among the cDNA sequences expressed in male strobili of $C$. japonica. RNA-Seq data from six libraries (Table S1) were used ${ }^{26}$. The genotype of $M S 1$ for these samples was determined based on phenotype and/or artificial crossing. cDNA sequences were screened using the following two criteria for MS1 candidate genes: (1) sequences expressed in male strobili but not in needles and inner bark and (2) sequences with twofold higher expression in male-fertile strobili compared to male-sterile strobili and vice versa were selected.

From the above procedure, 108 cDNA sequences were obtained as candidate genes of MS1. One of them, CJt020762, contained an SNP marker (AX-174139329; Hasegawa et al. $\left.{ }^{22}\right)$ located in the same position $(0 \mathrm{cM})$ as MS1 in the linkage map of the 'Fukushima-1' × 'Ooi-7' family. Furthermore, a 4-bp deletion causing a frameshift mutation was found in the coding region of CJt020762 (Figs. 1 and 2). This deletion was not detected in wildtype homozygous individuals but was detected heterozygously in three heterozygous individuals for MS1, and homozygously in two male-sterile individuals. Therefore, the presence of the $m s 1$ allele and this genetic mutation in the CJt020762 region were consistent with MS1. We hereafter focused our analysis on this gene.

CJt020762 codes for a lipid transfer protein gene containing a signal peptide, a plant lipid transfer protein domain, and a transmembrane domain in the coding region (Fig. 1). Furthermore, the glycosylphosphatidylinositol (GPI) anchor domain that contributes to fixing the protein on the outside of the plasma membrane was predicted at the C-terminus of CJt020762 (Figs. 1 and S1).

Genomic DNA sequence of MS1. Eight primers were designed based on the cDNA sequence of CJt020762 (Table S2), and the Sanger method was used to sequence the genomic DNA of 'Fukushima-1' (Table 1). The genome sequence of CJt020762 was 2,556-bp long, containing three coding sequence (CDS) regions (Figs. 1 and S2).

Haplotype composition of CJt020762 in breeding materials and individuals from natural forests. The haplotype sequences of CJt020762 of nine breeding materials with $m s 1$ and 74 individuals from 18 natural forest populations were determined. As a result, 49 haplotypes were detected from 83 individuals (Fig. 3 and Table S3). Of these haplotypes, only two (No. 38 (ms1-1) and No. 4 (ms1-2)) contained the deletion 
Ajigasawa20_1 (Haplotype No.1, WT)

Fukushima-1_1 (Haplotype No.38, 4-bp del)

Ooi-7_1 (Haplotype No.4, 30-bp del)

Ajigasawa20_1 (Haplotype No.1, WT)

Fukushima-1_1 (Haplotype No.38, 4-bp del)

Ooi-7_1
[ Signal peptide ]

1 MAAIVRAL I IFVLMAVAESVMTQQTDAAIDCTDPIVSLSPCLSYMTRSAKLSI

1 MAAIVRALI IFVLMAVAESVMTQQTDAAIDGTDPIVSLSPCLSYMTRSAKLSI

MAAIVRALI IFVLMAVAESVMTQQTDAAIDGTDPIVSLSPCLSYMTRSAKLSI

53

53

53

[ Plant lipid transfer protein domain ]

54 PDDDCCDSLATVINTHAGCLCELVSRDDFLGFPINQTITLSLPSTCDIQYPRE 106

54 PDDDCCDSLATVTPTQGACASSSPGTIFWAFPLIKPSLSRSLPPAIFNIRVNL 106

54 PDDDGCDSLATVINTHAGCLCELVSRDDFLGFPINQTITLSLPSTCDIQYPRE 106

Ajigasawa20_1 (Haplotype No.1, WT)

107 LDQGIVVLPPSSDGPVVQPGSNPPPRLFHPEAPSPALEAVPPVLEAPPMESVP

Fukushima-1_1 (Haplotype No.38, 4-bp del) 107 TSA

Ooi-7_1

(Haplotype No.4, 30-bp del) 107

LDQGIVVLPPSSDGPVVQPGSNPPPRLFHPEAPSPALEAVPPVLEAPPMESVP

[ Transmembrane domain ]

Ajigasawa20_1 (Haplotype No.1, WT)

160

Fukushima-1_1 (Haplotype No.38, 4-bp del) 109

DQPDDSPFPNVSIAGALFTQSLLRLFWGSLLTAILTVGYLH

$\uparrow$ [Potential GPI-Modification Site]

Figure 2. Amino-acid sequences for CJt020762. Underlines indicate the putative signal peptide, plant lipid transfer protein (LTP) domain and transmembrane domain. The blue boxes indicate the positions of eight conserved cysteine residues characteristic of LTPs. The red box indicates a potential modification site in the GPI (glycosylphosphatidylinositol) anchor domain.

\begin{tabular}{|c|c|c|c|c|}
\hline Tree ID & MS1 genotype & Indel type & Selected location & References \\
\hline Fukushima-1 & $m s 1 / m s 1$ & 4-bp & Yama-gun, Fukushima Prefecture, Japan & Igarashi et al. $^{5}$ \\
\hline Fukushima-2 & $m s 1 / m s 1$ & 4-bp & Yama-gun, Fukushima Prefecture, Japan & Igarashi et al. $^{5}$ \\
\hline Shindai-3 & $m s 1 / m s 1$ & 4-bp & Uonuma City, Niigata Prefecture, Japan & Ueuma et al. ${ }^{13}$, Saito et al. ${ }^{6}$ \\
\hline Shindai-11 & $m s 1 / m s 1$ & 4-bp & Uonuma City, Niigata Prefecture, Japan & Moriguchi et al. ${ }^{27}$ \\
\hline Shindai-12 & $m s 1 / m s 1$ & 4-bp & Uonuma City, Niigata Prefecture, Japan & Moriguchi et al. ${ }^{27}$ \\
\hline Tahara-1 & $m s 1 / m s 1$ & 4-bp & Hadano City, Kanagawa Prefecture, Japan & Saito $^{46}$ \\
\hline Naka-4 & $M s 1 / m s 1$ & 4-bp & Hadano City, Kanagawa Prefecture, Japan & Saito $^{17}$ \\
\hline Suzu-2 & $M s 1 / m s 1$ & 4-bp & Suzu City, Ishikawa Prefecture, Japan & Saito $^{17}$ \\
\hline Ooi-7 & $M s 1 / m s 1$ & $30-\mathrm{bp}$ & Haibara-gun, Shizuoka Prefecture, Japan & Saito $^{17}$ \\
\hline
\end{tabular}

Table 1. The breeding materials of Cryptomeria japonica with male-sterile gene (MS1) used in this study.

in the coding region. In haplotype No. 38, the frameshift mutation caused by the 4-bp deletion occurred in the middle of the plant lipid transfer protein domain (Fig. 2). On the other hand, in haplotype No. 4, the deletion of amino-acids occurred in the transmembrane domain due to the 30-bp deletion (Fig. 2). Furthermore, the modification site of the GPI anchor domain on the C-terminal side, which is important for transmembrane functioning, was lost after the 30-bp deletion (Figure S1). All individuals homozygous for $M S 1(\mathrm{~ms} 1 / \mathrm{ms} 1)$ (i.e., 'Fukushima-1', 'Fukushima-2', 'Shindai-3', 'Shindai-11', 'Shindai-12', and 'Tahara-1') had the homozygous haplotype No. 38 (Fig. 3 and Table 1), while individuals heterozygous for MS1 (Ms1/ms1) shown in Table 1 were heterozygous with haplotype No. 38 ('Naka-4', 'Suzu-2') or No. 4 ('Ooi-7') (Fig. 3 and Table 1). Haplotype No. 38 was absent from the 74 individuals from natural populations (Fig. 3 and Table S3). On the other hand, 17 individuals with haplotype No. 1, which is considered to be the ancestor of haplotype No. 38 ( $m s 1-1)$, were found in 13 natural populations (Ajigasawa, Mizusawa, Nibetsu, Yamanouchi, Donden, Bijodaira, Shimowada, Ashu, Tsuyama, Wakasugi, Azouji, Oninome and Yakushima; Figs. 3 and 4, Table S3). Haplotype No. $4(m s 1-2)$ with the 30-bp deletion was found in three individuals from the Ishinomaki natural population (Figs. 3 and 4, Table S3). Furthermore, five individuals with haplotype No. 2, which is considered to be the ancestor of haplotype No. 4, were found in three natural populations (Oki, Azouji and Oninome; Figs. 3 and 4, Table S3).

Amino-acid substitutions were found in six haplotypes from natural populations (Haplotype No. 36, 37, 39, 40, 41 and 42; Table S4). However, they were not detected in the breeding materials for $m s 1$.

\section{Discussion}

In this study, we searched for genetic mutations specific to C. japonica breeding materials with ms1 using RNASeq data. Based on the results, we identified CJt020762 as a causative gene for MS1. There is additional existing evidence supporting this finding. Firstly, CJt020762 contains the SNP marker (AX-174139329) mapped on $0 \mathrm{cM}$ from MS1 in the linkage map of the 'Fukushima-1' $\times$ 'Ooi-7' family ${ }^{22}$, indicating that CJt020762 was a gene in the 


\section{Ooi-7_1 [Msl/ms 1$]$}

Fukushima-1_1[msl/msl] Fukushima-1_2[ms $1 / \mathrm{ms} 1]$ Fukushima-2_1 [ms $1 / \mathrm{ms} 1]$ Fukushima-2_2[ms $1 / \mathrm{ms} 1]$ Shindai-3_1 [ms $1 / \mathrm{ms} l]$ Shindai-3_2[ $\left.\mathrm{msl}^{\mathrm{m}} \mathrm{ms} \mathrm{m}\right]$ Shindai-11_1[ms $1 / \mathrm{ms} 1]$ Shindai-11_2[ms $1 / \mathrm{ms} 1]$ Shindai-12_1 [ms $1 / \mathrm{ms} 1]$ Shindai-12_2[ms $1 / \mathrm{ms} I]$

Tahara-1_1 [msl/msl]

Tahara-1_2[msl/msl]

Naka-4_2[Msl/ms 1$]$

Suzu-2_2[Ms $1 / \mathrm{ms} 1]$

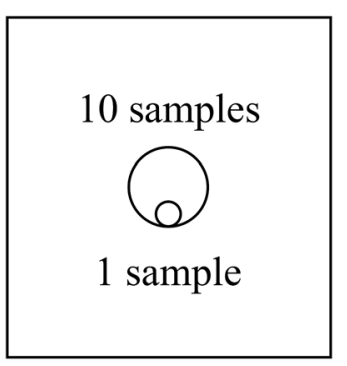

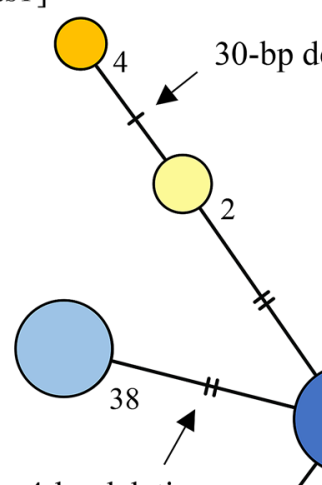

30-bp deletion in CDS

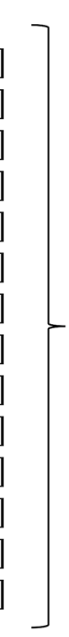

4-bp deletion $\begin{array}{r}\text { in CDS } \\ \hline\end{array}$

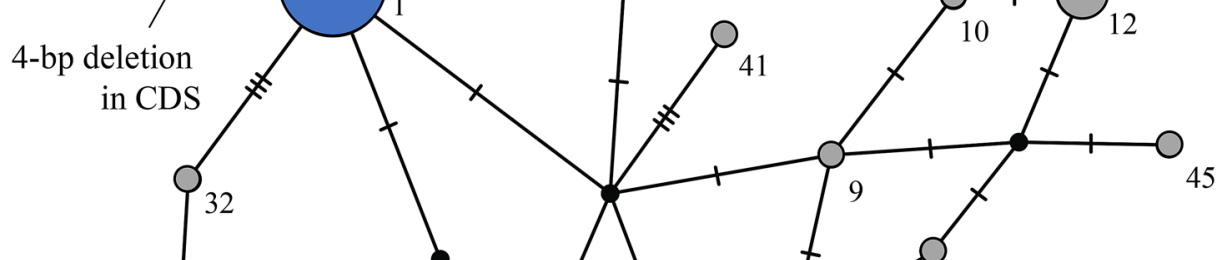

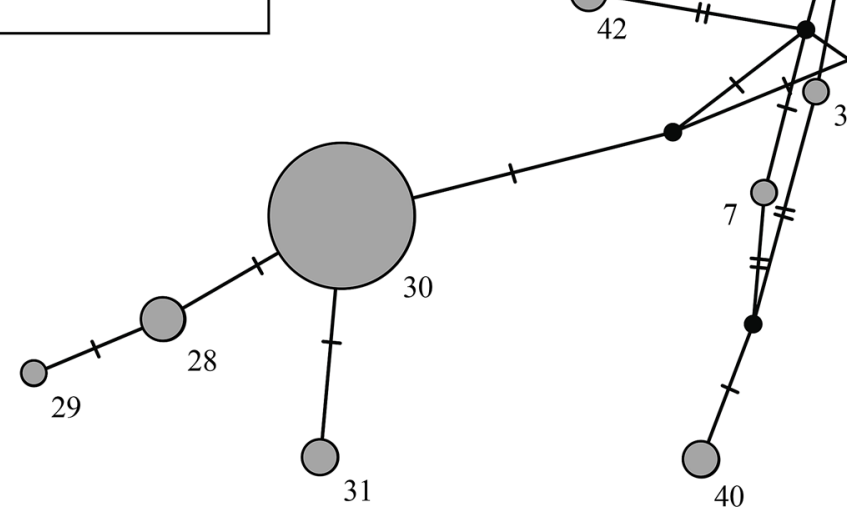
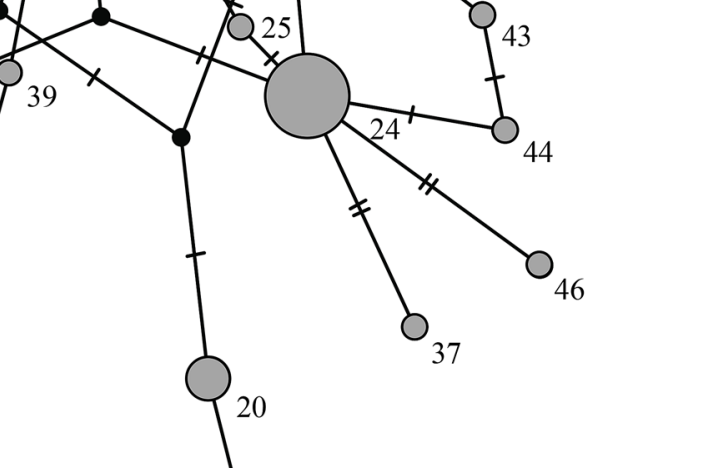

40

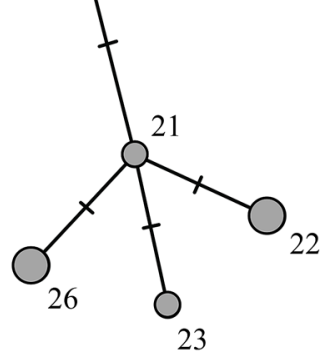

Figure 3. The haplotype network of CJt020762, based on 49 haplotypes determined from the 83 individuals including nine breeding materials with $m s 1$. Numbers indicate the haplotype numbers corresponding to Table S3. Haplotype names (e.g. Fukushima-1_1) indicate the haplotype of breeding materials with ms1. [ms1/ $m s 1]$ indicates homozygozity for $M S 1$, and [Ms1/ms1] indicates heterozygozity for $M S 1$. The node represents a haplotype. Node size represents haplotype frequency. The light blue segment represents the haplotype with a 4-bp deletion (No. 38); the blue segment represents the ancestor haplotype of the haplotype No. 38 (No. 1); the orange segment represents the haplotype with the 30-bp deletion (No. 4); the yellow segment represents the ancestor haplotype of the haplotype No. 4 (No. 2); and the grey segment represents the remaining 45 haplotypes. The horizontal line between nodes represents one SNP or one insertion/deletion (indel). 


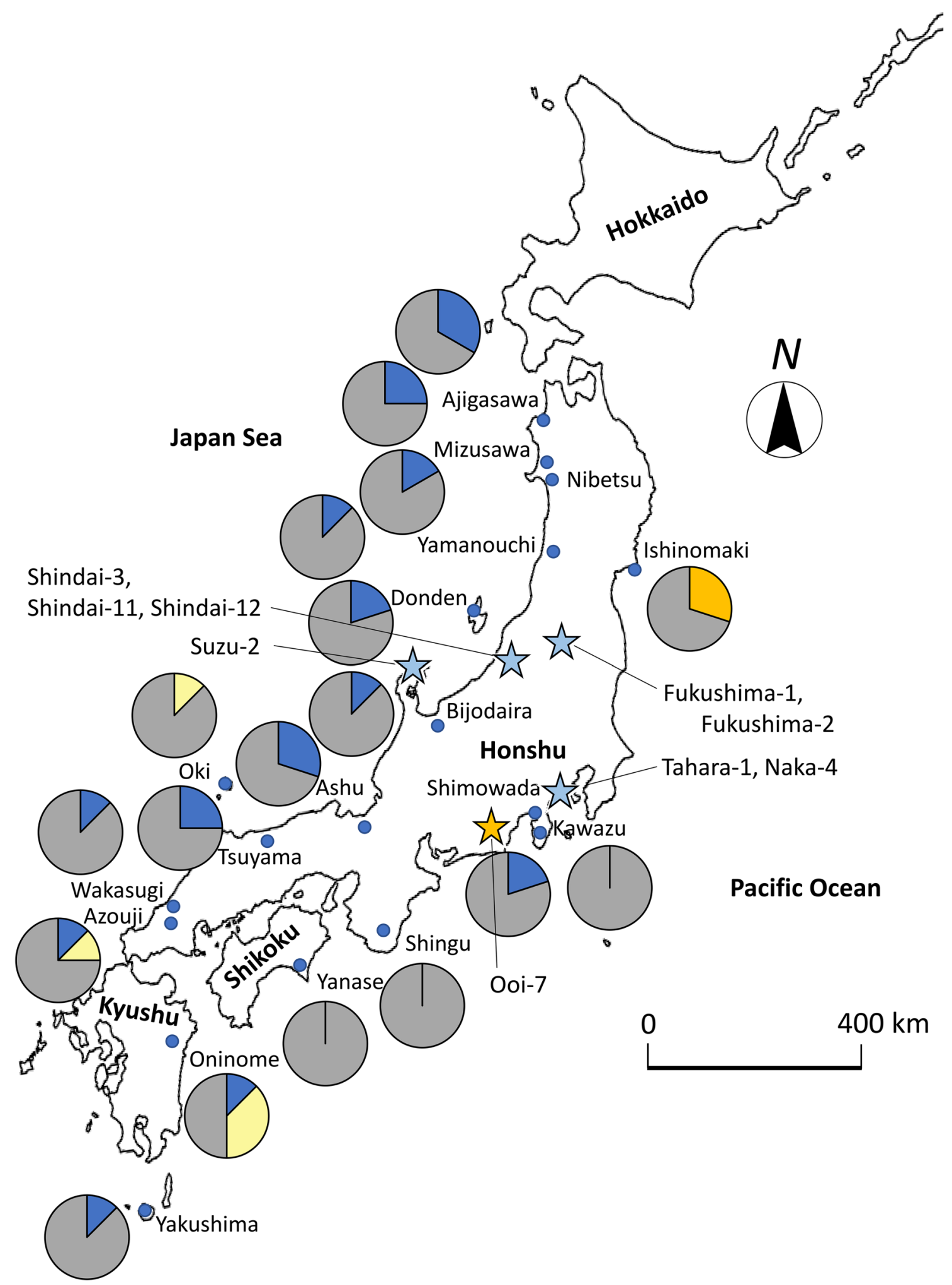

Figure 4. Geographical distribution of genomic DNA haplotypes of CJt020762 and breeding materials with $m s 1$. The light blue stars pinpoint locations where $m s 1$ breeding materials with a 4-bp deletion were selected; the orange star represents the location where 'Ooi-7' with a 30-bp deletion was selected; the blue segment represents the ancestor haplotype of the haplotype with a 4-bp deletion (No. 1); the orange segment represents the haplotype with a 30-bp deletion (No. 4); the yellow segment represents the ancestor haplotype of the haplotype with the 30-bp deletion (No. 2); and the grey segment represents the remaining 45 haplotypes. All haplotype No. correspond to those in Fig. 3 and Table S3. The map was generated using R3.5.1 (https://cran.r-project.org/bin/ windows/base/old/3.5.1/), QGIS3.14.15 (https://qgis.org/downloads/QGIS-OSGeo4W-3.14.15-1-Setup-x86_64. exe), and PowerPoint16.43 (https://www.microsoft.com) software. 
vicinity of MS1. Secondly, CJt020762 was expressed in male strobili, where it is active. Thirdly, both 4-bp and 30-bp deletions are expected to cause dysfunction in this particular gene: the 4-bp deletion mutation deleted the amino-acid sequence of the plant lipid transfer protein domain, causing a frameshift, while the 30-bp deletion mutation deleted 10 (63\%) amino-acids in the transmembrane domain (16 amino-acids). Furthermore, the modification site of the GPI anchor domain that contributes to fixing the protein to the outside of the plasma membrane was lost after the 30-bp deletion. These amino-acid sequence mutations are likely to result in the malfunction of the lipid transfer protein coded by CJt020762. Fourth, the cross between an individual with homozygous for the 4-bp deletion (ms1-1/ms1-1) and an individual with heterozygous for the 4-bp deletion (Ms1/ms1-1) resulted in 1:1 ratio of the male-sterile and male-fertile offspring in both of two families (i.e., 'Shindai-11' $\times$ 'Suzu-2' and 'Shindai-12' $\times$ 'Suzu-2') ' $^{27}$. Futhermore, the male-sterile and male-fertile offsprings always had the 4-bp deletion homozygously and the 4-bp deletion heterozygously in CJt020762, respectively ${ }^{27}$. The fifth piece of evidence suggesting that CJt020762 is the causative gene of MS1 comes from the mapping family 'Fukushima-1' $\times$ 'Ooi-7', where male-sterility occurred in one-half of offsprings ${ }^{22}$. Result of the DNA sequencing (Table S3) revealed that 'Fukushima-1' was homozygous for the 4-bp deletion haplotype ( $m s 1-1 / m s 1-1)$ and that 'Ooi-7' was heterozygous, with the 30-bp deletion and wild-type haplotype (Ms1/ms1-2). Therefore, it was clarified that even if CJt020762 is heterozygous for the 4-bp deletion haplotype and 30-bp deletion haplotype ( $m s 1-1 / m s 1-2)$, male-sterility occurs. Such a phenomenon occurs when CJt020762 codes a protein essential for pollen production and the protein function is lost due to the 4-bp deletion in the plant lipid transfer protein domain and the 30-bp deletion in the transmembrane domain. This result also indicates that both the plant lipid transfer protein domain and the transmembrane domain contained in CJt020762 is essential for pollen production in C. japonica. The sixth and final supporting evidence stems from the functional and structural similarity of CJt020762 with wheat male-sterility genes. In wheat male-sterility (Triticum aestivum), both ms 1 and $m s 5$ possess recessive inheritance and single locus control, hence male-sterility is caused by the failure of exine development during microspore formation ${ }^{28-31}$. MS1 in C. japonica and $m s 1$ and $m s 5$ in wheat have similar male-sterility phenotypes. In terms of protein structure, CJt020762 contains the signal peptide, plant lipid transfer protein domain, transmembrane domain, and the GPI anchor domain modification sites (Fig. 1). This structure is similar to $m s 1$ and $m s 5$ in wheat ${ }^{29-31}$. Furthermore, while the plant lipid transfer protein domain and transmembrane domain of CJt020762 may be necessary for pollen production in C. japonica, an analysis of mutant wheat revealed that both the lipid transfer protein domain and transmembrane domain of wheat $m s 1$ were necessary for pollen production ${ }^{30}$. Because of the similarities with wheat male-sterility genes, CJt020762 is thought to be essential for pollen production, as well as $m s 1$ and $m s 5$ in wheat, despite their respective aminoacid sequences being different to each other, with percentage identity ranging from 27.0 to 31.4 for the highest scoring segment pairs in BLASTP.

Based on above evidence, we propose that the CJt020762 section is the MS1 gene itself. We clarified the genomic DNA haplotypes of CJt020762 in 83 individuals, including nine breeding materials with $m s 1$ and 74 individuals from 18 natural forests. Eight individuals ('Fukushima-1', 'Fukushima-2', 'Shindai-3', 'Shindai-11', 'Shindai-12', 'Tahara-1', 'Naka-4', 'Suzu-2') with ms1-1 (haplotype No. 38) had a 4-bp deletion in the plant lipid transfer protein domain. Additionally, 'Ooi-7' with $m s 1-2$ (haplotype No. 4) had a 30-bp deletion in the transmembrane domain. These results suggest that these deletions were caused by independent two genetic mutation events. In the Ishinomaki natural forest, haplotype No. 4 with the 30-bp deletion (ms1-2) was found in 3/5 individuals. This result indicates that individuals with haplotype No. 4 may be more frequent in the Ishinomaki natural forest. The distance between the selected location of 'Shindai-3', 'Shindai-11', 'Shindai-12' and that of 'Tahara-1', 'Naka-4' was approximately $300 \mathrm{~km}$ and that between the selected location of 'Ooi-7' and the Ishinomaki natural forest was approximately $450 \mathrm{~km}$. Therefore, the single genetic deletion mutations were dispersed over hundreds of kilometers. Furthermore, haplotype No. $4(m s 1-2)$ was derived from ancestral haplotype No. 2, which is distributed in three southern populations (Oki, Azouji and Oninome). As haplotype No. 4 was found on the Pacific Ocean side in the central and northern regions of Honshu island ('Ooi-7' and Ishinomaki forest), it may have expanded further north along the Pacific side. Using markers developed during this study, a number of individuals with $m s 1$ can be selected from all over Japan in the future. Identifying the CJt020762 haplotype of these individuals and comparing it to the distribution of ancestral haplotypes will clarify the historical gene flow of $m s 1$.

The findings of this study suggest that CJt020762 is the causative gene for MS1 of C. japonica and contribute to the MAS of MS1 for breeding of male-sterile trees. Although the 16 individual trees with $m s 1$ have-until now-all been found in Japan ${ }^{5,11-14,16-18}$, it is necessary to select more individuals by MAS to obtain breeding materials in each region, a $C$. japonica had differentially adapted to unique environments in different regions of the Japanese archipelago, such as around the Japan Sea (heavy snow in winter) or along the Pacific coast (dry in winter) ${ }^{32}$. To definitely prove that CJt020762 is the causative gene for MS1, it is essential to show that male-sterility occurs by knocking out CJt020762 in C. japonica through genome editing in future research.

\section{Methods}

RNA-sequencing and MS1 annotation. To identify causative single nucleotide variations (SNVs) and/ or insertions or deletions (indels) in expressed genes, we analysed RNA-sequencing (RNA-Seq) data from six libraries (Table S1) from the mapping family of $M S 1$. These data have been reported in Ueno et al. ${ }^{25}$ and Wei et al. ${ }^{26}$ In this study, we focused on the levels of gene expression and selected candidate genes using CJ3006All by Wei $e a^{26}$ as a reference. We compared the expression levels among the 10 libraries. When multiple sequencing runs (sampling stages) were included in each library, we considered these runs as repetitions and labeled them as "_rep1," “_rep2," and so on (see a supplementary table by Wei et al. ${ }^{26}$ for details). Gene expression was quantified and compared by "kallisto ${ }^{33 \text { " and "sleuth }}{ }^{34}$," respectively, as described by Wei et $\mathrm{al}^{26}$. The candidate genes 
were selected with the following criteria: (1) expression levels (in transcripts per million [tpm]) in leaf or bark tissue (Ooi-7_rep1) < 10 and male strobilus tissue (S1s_rep2 and S3s_rep3) > 10; (2) expression levels (tpm) in fertile strobili (S3s_rep3) was more than twice compared to those in sterile strobili (S4s_rep3) and vice versa. We selected 108 candidate genes (Table S5) for MS1, which were then examined for SNVs and indels. All of the read mapping of the RNA-Seq was checked manually using an IGV (Integrative Genome Viewer) ${ }^{35}$ for homozygosity which was the only criteria after filtering by the expression levels before examining the variant effects. After we identified the causative functional mutation for MS1 from the RNA-Seq data (Figure S2) and verified the marker (AX-174139329) position on the linkage map, we examined the translated amino-acid sequences of the candidate gene (CJt020762) for the functional domain and annotated using SignalP-5.0 ${ }^{36}$, SMART (https://smart .embl.de/), PRED-TMBB2 ${ }^{37}$, and big-PI Plant Predictor ${ }^{38}$.

Plant materials and DNA extraction. This study used six breeding materials (trees) homozygous for MS1 ( $m s 1 / m s 1)$, three breeding materials heterozygous for MS1 (MS1/ms 1$)$, and 80 trees from 18 natural forests (Tables 1 and S6). Needle tissue was collected from all 89 trees; in natural forests, it was collected from an established scion garden through cutting. It was unknown whether the 89 trees in the natural forests were male-sterile or male-fertile. Genomic DNA was extracted from these needles using a modified CTAB method ${ }^{39}$.

Sanger sequencing of CJt020762. PCR was performed in $15 \mu \mathrm{L}$ volume containing approximately $60 \mathrm{ng}$ of genomic DNA of 'Fukushima-1' (Table 1), 1× Multiplex PCR Master Mix (Qiagen, Hilden, Germany), and $0.2 \mu \mathrm{M}$ of Primer_F3 and Primer_R (Figure S3 and Table S2). The thermal profile for the PCR was as follows: an initial denaturing step of $15 \mathrm{~min}$ at $95^{\circ} \mathrm{C}$, followed by 40 cycles of $30 \mathrm{~s}$ at $94{ }^{\circ} \mathrm{C}, 90 \mathrm{~s}$ at $63{ }^{\circ} \mathrm{C}$ and $90 \mathrm{~s}$ at $72{ }^{\circ} \mathrm{C}$, before a final elongation step at $72^{\circ} \mathrm{C}$ for 10 min using a GeneAmp 9700 PCR System (Applied Biosystems, PE Corp., Foster City, CA, USA). PCR products were purified using ExoSAP-IT (Affymetrix, Inc., Santa Clara, CA, USA). Direct sequencing was performed using the ABI PRISM BigDye Terminator version 3.1 Cycle Sequencing Kit (Applied Biosystems) on an ABI 3130 Genetic Analyser (Applied Biosystems). For sequencing, we also used additional internal primers (Primer_F2, Primer_F2-2, Primer_R2, Primer_R2-2, Primer_R2-3, Primer_R2-4) developed in this study (Figure S3 and Table S2). Sequence alignment was performed using CodonCode Aligner v.8.0.2 software (CodonCode Corporation, Dedham, MA, USA), followed by manual editing.

Amplicon sequencing of CJt020762. We developed seven primer pairs using the PCR suite software ${ }^{40}$ (Figure S3 and Table S2) using the genomic sequence of CJt020762 for 'Fukushima-1', following the Sanger method. We used these primer pairs and 89 individual trees (Tables 1 and S6) for the sequencing of whole CJt020762. Single-plex PCR was performed in $10 \mu \mathrm{L}$ volume containing approximately $30 \mathrm{ng}$ of genomic DNA, $1 \times$ Multiplex PCR Master Mix (Qiagen) and $0.2 \mu \mathrm{M}$ of each primer pair. The thermal profile for PCR was as follows: an initial denaturing step of $15 \mathrm{~min}$ at $95^{\circ} \mathrm{C}$, followed by 35 cycles of $30 \mathrm{~s}$ at $94{ }^{\circ} \mathrm{C}, 90 \mathrm{~s}$ at $66^{\circ} \mathrm{C}$ and $90 \mathrm{~s}$ at $72^{\circ} \mathrm{C}$ before a final elongation step at $72^{\circ} \mathrm{C}$ for $10 \mathrm{~min}$ by using a GeneAmp 9700 PCR System (Applied Biosystems). PCR products were pooled in equal volumes for each individual tree. Tag sequences for identifying individual trees were attached to the DNA fragments using the Access Array Barcode Library for Illumina Sequencers-384, Single Direction (Fluidigm Corporation, South San Francisco, CA, USA) and KAPA HiFi HotStart ReadyMix PCR Kit (KAPA BioSystems, Wilmington, MA, USA). PCR was performed in a $20 \mu \mathrm{L}$ volume containing $2 \mu \mathrm{L}$ of 20 -times diluted PCR products, $1 \times$ KAPA HiFi HS ReadyMix and $4 \mu \mathrm{L}$ of Access Array primers. The thermal profile for PCR was as follows: an initial denaturing step for $5 \mathrm{~min}$ at $95^{\circ} \mathrm{C}$, followed by 12 cycles of $15 \mathrm{~s}$ at $95^{\circ} \mathrm{C}, 30 \mathrm{~s}$ at $60^{\circ} \mathrm{C}$ and $60 \mathrm{~s}$ at $72^{\circ} \mathrm{C}$ before a final elongation step at $72^{\circ} \mathrm{C}$ for $3 \mathrm{~min}$ by using a GeneAmp 9700 PCR System (Applied Biosystems). After PCR, each DNA sample was purified using AMPure XP (Beckman Coulter, Brea, CA, USA). DNA concentration was determined using the Qubit fluorometer in the Qubit dsDNA HS Assay Kit (Thermo Fisher Scientific, Waltham, MA, USA). An equal amount of DNA from each sample was mixed to construct an amplicon sequencing library. The library was size-selected using BluePippin (1.5\% agarose cartridge, Sage Science, Beverly, MA, USA) under the range of 400-700 bp. The DNA concentration of the library was determined using the LightCycler 480 Real-Time PCR System (Roche, Basel, Switzerland) with KAPA Library Quantification Kit (KAPA Biosystems). Finally, the library was sequenced in $2 \times 251$-bp paired ends on MiSeq (Illumina, San Diego, CA, USA). Reads from each individual were automatically classified on MiSeq according to the tag sequences (DRR206539-DRR206627). After reads were cleaned using the Trimmomatic programme ${ }^{41}$, they were mapped onto the genomic sequence of CJt020762 from 'Fukushima-1' using the BWA mem algorithm ${ }^{42}$. Each mapping file (BAM) was imported into the Geneious software (Biomatters Ltd., Auckland, New Zealand) and possible SNP sites were manually checked. The consensus sequence was exported, then haplotype sequences of CJt020762 were estimated using the Phase software ${ }^{43,44}$. Because C. japonica is a diploid and has two haplotypes per individual, the haplotype ID was named as individual name_1 or individual name_2 (Table S3). Furthermore, to clarify phylogenetic relationships among the CJt020762 haplotypes, haplotype networks were created using the PopART software ${ }^{45}$. Six individual trees ('Ajigasawa02', 'Bijodaira03', 'Tsuyama03', 'Tsuyama07', 'Yakushima02', and 'Yamanouchi04') were excluded from the haplotype analysis as insufficient DNA sequences were obtained.

Received: 27 April 2020; Accepted: 21 December 2020

Published online: 15 January 2021 


\section{References}

1. Plomion, C. et al. Forest tree genomics: 10 achievements from the past 10 years and future prospects. Ann. For. Sci. 73, 77-103 (2016).

2. Holliday, J. A. et al. Advances in ecological genomics in forest trees and applications to genetic resources conservation and breeding. Mol. Ecol. 26, 706-717 (2017).

3. Agency Forestry. Statistical handbook of forest and forestry. Forestry Agency, Ministry of Agriculture, Forestry and Fisheries. pp. 8-9 (2014). (in Japanese)

4. Baba, K. \& Nakae, K. The national epidemiological survey of allergic rhinitis in 2008-comparison between 1998 and 2008 . Prog. Med. 28, 2001-2012 (2008) (in Japanese).

5. Igarashi, M., Watanabe, J., Ozawa, H., Saito, Y. \& Taira, H. The male sterile sugi (Cryptomeria japonica D. Don) was found in Fukushima Prefecture (I): selection of the search ground and identification of the male sterility. Tohoku J. For. Sci. 9, 86-89 (2004) (in Japanese with English abstract).

6. Saito, M. Breeding strategy for the pollinosis preventive cultivars of Cryptomeria japonica D Don. J. Jpn. For. Soc. 92, 316-323 (2010) (in Japanese with English abstract).

7. Taira, H., Saito, M. \& Furuta, Y. Inheritance of the trait of male sterility in Cryptomeria japonica. J. For. Res. 4, 271-273 (1999).

8. Yoshii, E. \& Taira, H. Cytological and genetical studies on male sterile sugi (Cryptomeria japonica D. Don), Shindai 1 and Shindai 5. J. Jpn. For. Soc. 89, 26-30 (2007) (in Japanese with English abstract).

9. Miyajima, D., Yoshii, E., Hosoo, Y. \& Taira, H. Cytological and genetic studies on male sterility in Cryptomeria japonica D. Don (Shindai 8). J. Jpn. For. Soc. 92, 106-109 (2010) (in Japanese with English abstract).

10. Moriguchi, Y. et al. A high-density linkage map with 2,560 markers and its application for the localization of the male-sterile genes $m s 3$ and $m s 4$ in Cryptomeria japonica D. Don. Tree Genet. Genomes 12, 57 (2016).

11. Taira, H., Teranishi, H. \& Kenda, Y. A case study of male sterility in sugi (Cryptomeria japonica). J. Jpn. For. Soc. 75, 377-379 (1993) (in Japanese with English abstract).

12. Takahashi, M. et al. Survey of male sterility on Sugi (Cryptomeria japonica D. Don) clones collected from Kanto Breeding Region and characteristics of two male-sterile clones. Bull. Natl. For. Tree Breed. Cent. 23, 11-36 (2007) (in Japanese with English abstract).

13. Ueuma, H., Yoshii, E., Hosoo, Y. \& Taira, H. Cytological study of a male-sterile Cryptomeria japonica that does not release microspores from tetrads. J. For. Res. 14, 123-126 (2009).

14. Yamada, H. \& Yamaguchi, K. Survey of male sterility of Sugi (Cryptomeria japonica D. Don) clones collected from Kansai Breeding Region and characteristics of the male-sterile clone. Appl. For. Sci. 8, 33-36 (2009) (in Japanese).

15. Ishii, K. et al. In vitro culture of various genotypes of male sterile Japanese cedar (Cryptomeria japonica D. Don). Plant Biotechnol. 28, 103-106 (2011).

16. Miura, S., Nameta, M., Yamamoto, T., Igarashi, M. \& Taira, H. Mechanisms of male sterility in four Cryptomeria japonica individuals with obvious visible abnormality at the tetrad stage. J. Jpn. For. Soc. 93, 1-7 (2011) (in Japanese with English abstract).

17. Saito, M., Aiura, H., Kato, A. \& Matsuura, T. Characterization of the cutting cultivar of Cryptomeria japonica D. Don, Zasshunbo, with a heterozygous male-sterility gene in a heterozygous state, selected from Toyama Prefecture. Japan. For. Genet. Tree Breed. 4, 45-51 (2015) (in Japanese with English abstract).

18. Kawai, Y., Kubota, M., Endoh, K. \& Isoda, K. Trial of efficient method for screening Cryptomeria japonica trees heterozygous for male-sterile gene by segregation of male-sterile seedlings derived from self-pollinated progeny. Bull. For. For. Prod. Res. Insti. 16, 265-266 (2017) (in Japanese).

19. Futamura, N., Igasaki, T., Saito, M., Taira, H. \& Shinohara, K. Comparison of fertile and sterile male gametogenesis in Cryptomeria japonica D. Don. Tree Genet. Genomes 15, 30 (2019).

20. Moriguchi, Y. et al. The construction of a high-density linkage map for identifying SNP markers that are tightly linked to a nuclearrecessive major gene for male sterility in Cryptomeria japonica D. Don. BMC Genomics 13, 95 (2012).

21. Moriguchi, Y. et al. A simple allele-specific PCR marker for identifying male-sterile trees: towards DNA marker-assisted selection in the Cryptomeria japonica breeding program. Tree Genet. Genomes 10, 1069-1077 (2014).

22. Hasegawa, Y. et al. Fine mapping of the male-sterile genes (MS1, MS2, MS3, and MS4) and development of SNP markers for marker-assisted selection in Japanese cedar (Cryptomeria japonica D. Don). PLoS ONE 13, e0206695 (2018).

23. Mishima, K. et al. Identification of novel putative causative genes and genetic marker for male sterility in Japanese cedar (Cryptomeria japonica D. Don). BMC Genomics 19, 277 (2018).

24. Tsubomura, M. et al. Development of simple DNA markers for selecting trees with the male-sterile gene of Cryptomeria japonica "Sosyun". J. Jpn. For. Soc. 101, 155-162 (2019) (in Japanese with English abstract).

25. Ueno, S. et al. Scanning RNA-Seq and RAD-Seq approach to develop SNP markers closely linked to MALE STERILITY 1 (MS1) in Cryptomeria japonica D. Don. Breed. Sci. 69, 19-29 (2019).

26. Wei, F. J. et al. Inspecting abundantly expressed genes in male strobili in sugi (Cryptomeria japonica D. Don) via a highly accurate cDNA assembly. bioRxiv. https://doi.org/10.1101/2020.04.21.054320 (2020).

27. Moriguchi, Y. et al. Marker-assisted selection of trees with MALE STERILITY 1 in Cryptomeria japonica D. Don. Forests 11, 734 (2020).

28. Tucker, E. J. et al. Molecular identification of the wheat male fertility gene Ms1 and its prospects for hybrid breeding. Nat. Commun. 8, 869 (2017).

29. Wang, Z. et al. Poaceae-specific MS1 encodes a phospholipid-binding protein for male fertility in bread wheat. Proc. Natl. Acad. Sci. U. S. A. 21, 12614-12619 (2017).

30. Kouidri, A. et al. Wheat TaMs1 is a glycosylphosphatidylinositol-anchored lipid transfer protein necessary for pollen development. BMC Plant Biol. 18, 332 (2018).

31. Pallotta, M. A. et al. Wheat $m s 5$ male-sterility is induced by recessive homoeologous A and D genome non-specific Lipid Transfer Proteins. Plant J. 99, 673-685 (2019).

32. Tsumura, Y. et al. Genetic differentiation and evolutionary adaptation in Cryptomeria japonica. G3-Genes Genomes Genet. 4, 2389-2402 (2014).

33. Bray, N. L., Pimentel, H., Melsted, P. \& Pachter, L. Near-optimal probabilistic RNA-seq quantification. Nat. Biotechnol. 34, 525-527 (2016).

34. Pimentel, H., Bray, N. L., Puente, S., Melsted, P. \& Pachter, L. Differential analysis of RNA-seq incorporating quantification uncertainty. Nat. Methods 14, 687-690 (2017).

35. Robinson, J. T. et al. Integrative genomics viewer. Nat. Biotechnol. 29, 24-26 (2011).

36. Almagro Armenteros, J. J. et al. SignalP 5.0 improves signal peptide predictions using deep neural networks. Nat. Biotechnol. 37, 420-423 (2019).

37. Tsirigos, K. D., Elofsson, A. \& Bagos, P. G. PRED-TMBB2: improved topology prediction and detection of beta-barrel outer membrane proteins. Bioinformatics 32, i665-i671 (2016).

38. Eisenhaber, B. et al. Glycosylphosphatidylinositol lipid anchoring of plant proteins. Sensitive prediction from sequence- and genome-wide studies for Arabidopsis and rice. Plant Physiol. 133, 1691-1701 (2003).

39. Murray, M. \& Thompson, W. F. Rapid isolation of high molecular weight plant DNA. Nucleic Acids Res. 8, 4321-4325 (1980). 
40. van Baren, M. J. \& Heutink, P. The PCR suite. Bioinformatics 20, 591-593 (2004)

41. Bolger, A. M., Lohse, M. \& Usadel, B. Trimmomatic: a flexible trimmer for illumina sequence data. Bioinformatics 30, 2114-2120 (2014).

42. Li, H. Aligning sequence reads, clone sequences and assembly contigs with BWA-MEM. arXiv 1303.3997v2 (2013).

43. Stephens, M., Smith, N. J. \& Donnelly, P. A new statistical method for haplotype reconstruction from population data. Am. J. Hum. Genet. 68, 978-989 (2001).

44. Stephens, M. \& Scheet, P. Accounting for decay of linkage disequilibrium in haplotype inference and missing-data imputation. Am. J. Hum. Genet. 76, 449-462 (2005).

45. Leigh, J. W. \& Bryant, D. POPART: full-feature software for haplotype network construction. Methods Ecol. Evol. 6, 1110-1116 (2015).

46. Saito, H. Development and verification of precision of a simple screening method for male sterility in seedling production of Japanese cedar (Cryptomeria japonica D. Don). J. Jpn. For. Soc. 102, 311-316 (2020) (in Japanese with English abstract).

\section{Acknowledgements}

The authors thank Yasuyuki Komatsu and Nozomi Ohmiya for their assistance with laboratory work, Yukiko Ito, Hiroshi Saito, and Akira Ogura for providing breeding materials for this study. This work was supported by research Grants \#201421 from the Forestry and Forest Products Research Institute, Grant-in-Aid from the Program for Promotion of Basic and Applied Researches for Innovations in Bio-oriented Industry (No.28013B), the project of the NARO Bio-oriented Technology Research Advancement Institution (Research program on development of innovative technology, No.28013BC), and NIBB Collaborative Research Program (16-403, 17-405 and 18-408). Computations were partially performed on the supercomputer of AFFRIT, MAFF, Japan.

\section{Author contributions}

Y.H., S.U., A.M., K.U., T.U., M.K., S.S., Y.T. and Y.M. conceived and designed the experiments. Y.H., S.U., A.M., T.H., T.B. and K.Y. and Y.M. performed the experiments. Y.H., S.U., F.W., T.F., M.K., S.S. and Y.M. analysed the data. Y.H., S.U. and Y.M. wrote the paper.

\section{Competing interests}

The authors declare no competing interests.

\section{Additional information}

Supplementary Information The online version contains supplementary material available at https://doi. org/10.1038/s41598-020-80688-1.

Correspondence and requests for materials should be addressed to S.U. or Y.M.

Reprints and permissions information is available at www.nature.com/reprints.

Publisher's note Springer Nature remains neutral with regard to jurisdictional claims in published maps and institutional affiliations.

(c) (i) Open Access This article is licensed under a Creative Commons Attribution 4.0 International License, which permits use, sharing, adaptation, distribution and reproduction in any medium or format, as long as you give appropriate credit to the original author(s) and the source, provide a link to the Creative Commons licence, and indicate if changes were made. The images or other third party material in this article are included in the article's Creative Commons licence, unless indicated otherwise in a credit line to the material. If material is not included in the article's Creative Commons licence and your intended use is not permitted by statutory regulation or exceeds the permitted use, you will need to obtain permission directly from the copyright holder. To view a copy of this licence, visit http://creativecommons.org/licenses/by/4.0/.

(c) The Author(s) 2021 